Bull. Korean Math. Soc. 51 (2014), No. 4, pp. 1135-1144

http://dx.doi.org/10.4134/BKMS.2014.51.4.1135

\title{
FINITE GROUPS ALL OF WHOSE MAXIMAL SUBGROUPS ARE SB-GROUPS
}

\author{
Pengfei Guo, Junxin Wang, and Hailiang Zhang
}

\begin{abstract}
A finite group $G$ is called a SB-group if every subgroup of $G$ is either $s$-quasinormal or abnormal in $G$. In this paper, we give a complete classification of those groups which are not SB-groups but all of whose proper subgroups are SB-groups.
\end{abstract}

\section{Introduction}

All groups considered here are assumed to be finite, and all notations employed are standard.

Let $\Sigma$ be an abstract group theoretical property. If all proper subgroups of a group $G$ have the property $\Sigma$ but $G$ does not have the property $\Sigma$, then $G$ is called a minimal non- $\Sigma$-group. One of the hottest topics in group theory is to determinate the structure of minimal non- $\Sigma$-groups and many meaningful results about this topic have been obtained. For example, O. J. Schmidt [12] determined the structure of minimal non-nilpotent groups, K. Doerk [4] elucidated the structure of minimal non-supersolvable groups, and J. G. Thompson [14] gave the classification of nonsolvable groups all of whose local subgroups are solvable. These achievements have greatly pushed forward the developments of group theory. The more new results can be referred to refs. [7, 8, 9, 11, 13, 17].

The aim of the present article is to study the structures of some minimal non$\Sigma$-groups. Recall that a subgroup $H$ of a group $G$ is an abnormal subgroup if $x \in\left\langle H, H^{x}\right\rangle$ for every $x \in G$. A subgroup $H$ of a group $G$ is said to be quasinormal in $G$ if $H K=K H$ for any subgroup $K$ of $G$, and $H$ is said to be $s$-quasinormal in $G$ if $H S=S H$ for any Sylow subgroup $S$ of $G$. A. Fattahi [5]

Received September 9, 2013.

2010 Mathematics Subject Classification. Primary 20D10, 20 E34.

Key words and phrases. SB-group, minimal non-SB-group, supersolvable group, power automorphism.

This work was partially supported by the National Natural Science Foundation of China (No. 11371237), the National Natural Science Foundation of China (No. 11171169), Jiangsu Overseas Research \& Training Program for University Prominent Young \& Middle-Aged Teachers and Presidents, Qing Lan Project of Jiangsu Province, and " 333 " Project of Jiangsu Province. 
called a group $G$ a B-group if every primary subgroup (i.e., subgroup of primepower order) of $G$ is either normal or abnormal in $G$, and obtained the structure of B-groups. Q. H. Zhang [16] studied the groups in which every subgroup is either $s$-quasinormal (resp. quasinormal) or abnormal, and gave the structures of the two kinds of groups. In this paper, we call such groups SB-groups (resp. QB-groups), and call a group $G$ a minimal non-SB-group (resp. minimal nonQB-group, minimal non-B-group) if every proper subgroup of $G$ is a SB-group (resp. QB-group, B-group) but $G$ itself is not. The complete classifications of the minimal non-SB-groups, minimal non-QB-groups and minimal non-Bgroups are shown as follows.

Theorem 1.1. Let $p, q$ and $r$ be distinct primes. Then a group $G$ is a minimal non-SB-group if and only if $G$ is one of the following types:

(I) $G=\left\langle x, y \mid x^{p}=y^{q^{n}}=1, y^{-1} x y=x^{i}\right\rangle$, where $p \equiv 1(\bmod q), n \geq 2$, $i^{q} \not \equiv 1(\bmod p), i^{q^{2}} \equiv 1(\bmod p)$ with $1<i<p$;

(II) $G=\left\langle x, y \mid x^{p q}=y^{q}=1, y^{-1} x y=x^{i}\right\rangle$, where $p \equiv 1(\bmod q), i \equiv$ $1(\bmod q), i^{q} \equiv 1(\bmod p)$ with $1<i<p$;

(III) $G=\left\langle x, y \mid x^{4 p}=1, y^{2}=x^{2 p}, y^{-1} x y=x^{-1}\right\rangle$, where $p \neq 2$;

(IV) $G=\left\langle x, y, z \mid x^{p}=y^{q^{n-1}}=z^{q}=1, y^{-1} x y=x^{i},[x, z]=1,[y, z]=1\right\rangle$, where $p \equiv 1(\bmod q), n \geq 3, i^{q} \equiv 1(\bmod p)$ with $1<i<p$;

(V) $G=\langle x, y, z| x^{p}=y^{q^{n-1}}=z^{q}=1, y^{-1} x y=x^{i},[x, z]=1,[y, z]=$ $\left.y^{q^{n-2}}\right\rangle$, where $p \equiv 1(\bmod q)$, $i^{q} \equiv 1(\bmod p)$ with $1<i<p, n \geq 3$, and $n \geq 4$ if $q=2$;

(VI) $G=P \rtimes Q$, where $P=\langle a, b\rangle$ is an elementary abelian $p$-group of order $p^{2}$, and $Q=\langle y\rangle$ is cyclic of order $q^{r}$. The action of $Q$ on $P$ is defined by $a^{y}=a^{i}, b^{y}=b^{j}, p \equiv 1(\bmod q)$, and $r \geq 1$, where $i$ is a primitive $q$-th root of unity modulo $p, i \neq \equiv j(\bmod p)$ with $0<i, j<p$;

(VII) $G=P \rtimes Q$, where $Q=\langle y\rangle$ is cyclic of order $q^{r}>1$, with $q \nmid p-1$, and $P$ is an irreducible $Q$-module over the field of $p$ elements with kernel $\left\langle y^{q}\right\rangle$ in $Q$;

(VIII) $G=P \rtimes Q$, where $P$ is a non-abelian special $p$-group of rank $2 m$, the order of $p$ modulo $q$ being $2 m, Q=\langle y\rangle$ is cyclic of order $q^{r}>1$, y induces an automorphism in $P$ such that $P / \Phi(P)$ is a faithful and irreducible $Q$-module, and $y$ centralizes $\Phi(P)$. Furthermore, $|P / \Phi(P)|=p^{2 m}$ and $\left|P^{\prime}\right| \leq p^{m}$;

(IX) $G=P \rtimes Q$, where $P=\left\langle a_{0}, a_{1}, \ldots, a_{q-1}\right\rangle$ is an elementary abelian p-group of order $p^{q}, Q=\langle y\rangle$ is cyclic of order $q^{r}, q$ is the highest power of $q$ dividing $p-1$ and $r>1$. The action of $Q$ on $P$ is defined by $a_{j}^{y}=a_{j+1}$ for $0 \leq j<q-1$ and $a_{q-1}^{y}=a_{0}^{i}$, where $i$ is a primitive $q$-th root of unity modulo $p$

(X) $G=\left\langle a, b \mid a^{p}=b^{q^{m}}=1, b^{-1} a b=a^{i}\right\rangle$, where $p>r, q>r, p \equiv$ $1(\bmod r),\left(p, q r^{m}(i-1)\right)=1, i^{r} \equiv 1(\bmod p)$ and $i^{q} \not \equiv 1(\bmod p)$ with $1<i<p$

(XI) $G=\left\langle a, b \mid a^{p}=b^{q^{m} r}=1, b^{-1} a b=a^{i}\right\rangle$, where $p>q>r, p \equiv 1(\bmod q)$, $\left(p, q^{m} r(i-1)\right)=1, i^{r} \not \equiv 1(\bmod p)$ and $i^{q} \equiv 1(\bmod p)$ with $1<i<p$; 
FINITE GROUPS ALL OF WHOSE MAXIMAL SUBGROUPS ARE SB-GROUPS 1137

(XII) $G=\left\langle a, b \mid a^{p}=b^{q r}=1, b^{-1} a b=a^{i}\right\rangle$, where $p>q>r, p \equiv$ $1(\bmod q r),(p, q r(i-1))=1, i$ is a primitive $q r$-th root of unity modulo $p$ with $1<i<p$.

Theorem 1.2. The minimal non-QB-groups are exactly the groups of types (I) to (XII) of Theorem 1.1, with $P$ is also a quasi-Hamilton group in the case of groups of type (VIII).

Theorem 1.3. The minimal non-B-groups are exactly the groups of types (I) to (IV), (VI) to (XII) of Theorem 1.1, with $p=2, q=3$ and $m=1$ in the case of groups of type (VIII).

\section{Preliminary results}

We collect some lemmas which will be frequently used in the following deduction.

Lemma 2.1 ([16, Theorem 2]). If a group $G$ is a $S B$-group, then $G$ is supersolvable.

Lemma 2.2 ([4]). Let $G$ be a minimal non-supersolvable group. Then

(1) $G$ has a unique normal Sylow p-subgroup $P$;

(2) $P / \Phi(P)$ is a minimal normal subgroup of $G / \Phi(P)$, and $P / \Phi(P)$ is noncyclic;

(3) If $p \neq 2$, then the exponent of $P$ is $p$;

(4) If $P$ is non-abelian and $p=2$, then the exponent of $P$ is at most 4;

(5) If $P$ is abelian, then the exponent of $P$ is $p$.

Lemma 2.3 ([16, Theorem 3]). A group $G$ is a $S B$-group if and only if $G$ is precisely one of the following:

I) $G$ is a nilpotent group;

II) $G=H P$, where $H$ is a normal abelian $p^{\prime}$-Hall subgroup of $G, P=$ $\langle x\rangle \in \operatorname{Syl}_{p}(G),\left\langle x^{p}\right\rangle=O_{p}(G)=Z(G), x$ induces a fixed-point-free power automorphism of order $p$ on $H$, and $p$ is the smallest prime factor of $|G|$.

Lemma 2.4. Let $G$ be a minimal non-SB-group. Then $G$ has a normal Sylow subgroup and $|\pi(G)| \leq 3$.

Proof. By assumption, every proper subgroup of $G$ is a SB-group, and $G$ is supersolvable or minimal non-supersolvable by Lemma 2.1. Lemma 2.2 implies that $G$ is solvable. Let $\mathcal{A}=\left\{P_{1}, P_{2}, \ldots, P_{s}\right\}$ be a Sylow basis, where $P_{i}$ is a Sylow $p_{i}$-subgroup of $G$ and $p_{1}<p_{2}<\cdots<p_{s}$. Suppose that $s \geq 4$.

Since $P_{i} P_{s}<G, P_{i} P_{s}$ is supersolvable. Thus, $P_{s}$ is normalized by each $P_{i}(i=1,2, \ldots, s)$, and so $P_{s} \unlhd G$. Since $P_{s}$ has a complement which must be supersolvable, it follows that $G$ has a Sylow tower.

Without loss of generality, we suppose that $s=4$. If $P_{1}$ is non-cyclic, then by Lemma 2.3, $P_{1} P_{2} P_{3}, P_{1} P_{2} P_{4}$ and $P_{1} P_{3} P_{4}$ must be nilpotent, and so $G$ is nilpotent, a contradiction. Hence $P_{1}$ is cyclic. If one of $P_{1} P_{2} P_{3}, P_{1} P_{2} P_{4}$ and 
$P_{1} P_{3} P_{4}$ is nilpotent, then by Lemma 2.3 , all of them are nilpotent, and so $G$ is nilpotent, a contradiction. Thus, each of $P_{1} P_{2} P_{3}, P_{1} P_{2} P_{4}$ and $P_{1} P_{3} P_{4}$ is not nilpotent. By Lemma 2.3, $P_{2} P_{3}, P_{2} P_{4}$ and $P_{3} P_{4}$ are all abelian, and every element of $P_{1}$ acting on $P_{2} P_{3}, P_{2} P_{4}$ and $P_{3} P_{4}$ are fixed-point-free power automorphisms of order $p$. Clearly, $P_{2} P_{3} P_{4}$ is abelian, and every element of $P_{1}$ acting on $P_{2} P_{3} P_{4}$ is a fixed-point-free power automorphism of order $p$, and so $G$ is a SB-group, a contradiction. Hence $|\pi(G)| \leq 3$.

Lemma 2.5 ([10, 13.4.3]). Let $\alpha$ be a power automorphism of an abelian group $A$. If $A$ is a p-group of finite exponent, then there is a positive integer $l$ such that $a^{\alpha}=a^{l}$ for all $a$ in $A$. If $\alpha$ is nontrivial and has order prime to $p$, then $\alpha$ is fixed-point-free.

Lemma 2.6 ([6, Lemma 2.9]). If a p-group $G$ of order $p^{n+1}$ has a unique noncyclic maximal subgroup, then $G$ is isomorphic to one of the following groups:

(I) $C_{p^{n}} \times C_{p}=\left\langle a, b \mid a^{p^{n}}=b^{p}=1,[a, b]=1\right\rangle$, where $n \geq 2$;

(II) $M_{p^{n+1}}=\left\langle a, b \mid a^{p^{n}}=b^{p}=1,[a, b]=a^{p^{n-1}}\right\rangle$, where $n \geq 2$, and $n \geq 3$ if $p=2$.

Lemma 2.7 ([16, Corollary 2]). A group $G$ is a $Q B$-group if and only if $G$ is precisely one of the following:

I) $G$ is a quasi-Hamilton group;

II) The group as listed in Lemma $2.3 \mathrm{II})$.

Lemma 2.8 ([16, Corollary 3]). A group $G$ is an B-group if and only if $G$ is precisely one of the following:

I) $G$ is a Dedekind group;

II) The group as listed in Lemma $2.3 \mathrm{II})$.

Lemma 2.9 ([18]). A p-group $G$ is a quasi-Hamilton group if and only if $G$ is either a Hamilton group or $G$ has an abelian normal subgroup $A$ such that $G / A$ is cyclic, and there exist a positive integer $s$ and an element $g$ of $G$ such that $G=\langle A, g\rangle, a^{g}=a^{1+p^{s}}$ for any $a \in G, s \geq 2$ if $p=2$.

\section{The proof of Theorem 1.1}

Proof. If $G$ is a minimal non-SB-group, then by Lemma $2.4,|\pi(G)| \leq 3$.

We first consider the case of $|\pi(G)|=2$, and assume that $G=P Q$ with $P \unlhd G$ and $Q \not G$ by Lemma 2.4, where $P \in \operatorname{Syl}_{p}(G)$ and $Q \in \operatorname{Syl}_{q}(G)$.

Assume that neither $P$ nor $Q$ is cyclic. Let $P_{1}$ be an arbitrary maximal subgroup of $P$, and let $Q_{1}$ and $Q_{2}$ be two distinct maximal subgroups of $Q$. By assumption, $P Q_{1}$ and $P Q_{2}$ are SB-groups. Lemma 2.3 implies that $P_{1}$ is normal in $G=\left\langle P, Q_{1}, Q_{2}\right\rangle$. For two distinct maximal subgroups $A$ and $B$ of $P, A Q$ and $B Q$ are SB-groups by assumption. By Lemma 2.3, $Q$ is normal in $G=\langle A, B, Q\rangle$, a contradiction. Hence either $P$ or $Q$ is cyclic.

Now we divide the situation into the following four cases to be discussed. 
(1) Assume that $P$ and $Q$ are both cyclic, and let $P=\langle x\rangle$ and $Q=\langle y\rangle$ with $|x|=p^{m}$ and $|y|=q^{n}$. In this case, $y^{-1} x y=x^{i}$ with $i^{q^{n}} \equiv 1\left(\bmod p^{m}\right)$, $p \equiv 1(\bmod q), 1<i<p^{m}$ and $\left(p^{m}, q^{n}(i-1)\right)=1$. By assumption, $P\left\langle y^{q}\right\rangle$ and $\left\langle x^{p}\right\rangle Q$ are both SB-groups. Clearly, $\left\langle y^{q}\right\rangle$ is not normal in $G$, and $\left\langle y^{q^{2}}\right\rangle$ is normal in $\langle x\rangle\left\langle y^{q}\right\rangle$. We have $\left(y^{q}\right)^{-1} x y^{q}=x^{i^{q}} \neq x$ and $\left(y^{q^{2}}\right)^{-1} x y^{q^{2}}=x^{i^{q^{2}}}=x$. Hence $i^{q} \not \equiv 1\left(\bmod p^{m}\right), y^{q}$ induces a power automorphism of order $q$ in $P$, and every proper subgroup of $\left\langle y^{q}\right\rangle$ is normal in $G$. If $x^{p} \neq 1$, then by Lemma 2.5, $\left\langle x^{p}\right\rangle\left\langle y^{q}\right\rangle \neq\left\langle x^{p}\right\rangle \times\left\langle y^{q}\right\rangle$. However, we have $\left\langle x^{p}\right\rangle\left\langle y^{q}\right\rangle=\left\langle x^{p}\right\rangle \times\left\langle y^{q}\right\rangle$ since $\left\langle y^{q}\right\rangle$ is normal in $\left\langle x^{p}\right\rangle\langle y\rangle$, a contradiction. So $G$ is of type (I).

(2) Assume that $P$ is cyclic, $Q$ is non-cyclic and $p>q$. If $Q$ has two distinct non-cyclic maximal subgroups $Q_{1}$ and $Q_{2}$, then by Lemma 2.3, $P Q_{1}=P \times Q_{1}$ and $P Q_{2}=P \times Q_{2}$. Hence $Q=Q_{1} Q_{2}$ is normal in $G$, a contradiction. Therefore, all maximal subgroups of $Q$ are cyclic or $Q$ has a unique non-cyclic maximal subgroup.

Case 1. All maximal subgroups of $Q$ are cyclic. It is clear that $Q$ is either an elementary abelian group of order $q^{2}$ or the quaternion group of order 8 .

If $Q$ is elementary abelian, then we let $P=\langle z\rangle, Q=\langle a, b| a^{q}=b^{q}=$ $1,[a, b]=1\rangle, a^{-1} z a=z^{i}, b^{-1} z b=z^{j}$, where $i^{q} \equiv j^{q} \equiv 1(\bmod p), 1 \leq i, j<p$, $|z|=p^{m}$ with $m \geq 1$. If $z^{p} \neq 1$, then $\left\langle z^{p}\right\rangle Q=\left\langle z^{p}\right\rangle \times Q$ by Lemma 2.3. Clearly, there exists a nontrivial power automorphism that $a$ or $b$ acts on $P$ by conjugation. Without loss of generality, we assume that $a$ acting on $P$ by conjugation is nontrivial. By Lemma 2.5, $\left\langle z^{p}\right\rangle\langle a\rangle \neq\left\langle z^{p}\right\rangle \times\langle a\rangle$, which contradicts the fact that $\left\langle z^{p}\right\rangle Q=\left\langle z^{p}\right\rangle \times Q$. Thus, $z^{p}=1$. If $C_{G}(P)=P$, then $G / C_{G}(P)$ is an elementary abelian group of order $q^{2}$. However, $G / C_{G}(P) \lesssim \operatorname{Aut}(P)$, and $\operatorname{Aut}(P)$ is cyclic, a contradiction. Therefore one of $a$ and $b$ is contained in $C_{G}(P)$ but the other is not. We can let $C_{G}(P)=\langle x\rangle, y^{-1} x y=x^{i}$, where $x=z b$ is a generator of $C_{G}(P),|x|=p q, y=a, i \equiv 1(\bmod q)$ and $i^{q} \equiv 1(\bmod p)$. So $G$ is of type (II).

If $Q$ is the quaternion group of order 8 , then we let $Q=\langle a, b| a^{4}=1, b^{2}=$ $\left.a^{2}, b^{-1} a b=a^{-1}\right\rangle$ and $P=\langle z\rangle$. With similar arguments as above, there exists a nontrivial power automorphism that $a$ or $b$ acts on $P$ by conjugation, and $z^{p}=1$. We can assume that $a$ acting on $P$ by conjugation is nontrivial. Since $P\langle a\rangle$ is a SB-group, $\left\langle a^{2}\right\rangle \leq C_{G}(P)$. If $C_{G}(P)=P \times\left\langle a^{2}\right\rangle$, then $G / C_{G}(P)$ is an elementary abelian group of order 4 , which contradicts the fact that $G / C_{G}(P) \lesssim \operatorname{Aut}(P)$, and $\operatorname{Aut}(P)$ is cyclic. Therefore, $C_{G}(P)$ must contain an element of order 4 , and let $C_{G}(P)=\langle x\rangle$ be a cyclic group of order $4 p$, where $x$ is a generator of $C_{G}(P)$. Surely, $G$ has an element $y$ of order 4 such that $y \neq x^{p}$, and let $y^{-1} x y=x^{i}$, where $i \not \equiv 1(\bmod 4 p)$. Since $\left(y^{2}\right)^{-1} x y^{2}=x^{i^{2}}=x$, we have $i^{2} \equiv 1(\bmod 4 p)$. By calculations, $G=\left\langle x, y \mid x^{4 p}=1, y^{2}=x^{2 p}, y^{-1} x y=x^{-1}\right\rangle$. So $G$ is of type (III).

Case 2. Let $P=\langle x\rangle$, and let $Q$ be as in Lemma 2.6 (I) with $|Q|=q^{n}$. That is, $Q=\left\langle y, z \mid y^{q^{n-1}}=z^{q}=1,[y, z]=1\right\rangle$, where $n \geq 3$. Then $Q$ has the maximal subgroups $H=\langle y\rangle, K_{0}=\left\langle y^{q}, z\right\rangle$ and $K_{s}=\left\langle y^{q}, z y^{s}\right\rangle=\left\langle z y^{s}\right\rangle$ with 
$s=1, \ldots, q-1$, where $K_{0}$ is the unique non-cyclic maximal subgroup of $Q$. By Lemma 2.3, $P K_{0}=P \times K_{0}$. Clearly $P H \neq P \times H$ and $z \in Z(G)$. Since $P H$ is a SB-group, $y$ induces a power automorphism of order $q$ in $P$. With similar arguments used in Case 1 , we can prove $x^{p}=1$. Hence $G=\langle x, y, z| x^{p}=$ $\left.y^{q^{n-1}}=z^{q}=1, y^{-1} x y=x^{i},[x, z]=1,[y, z]=1\right\rangle$, where $n \geq 3, p \equiv 1(\bmod q)$, $i^{q} \equiv 1(\bmod p)$ with $1<i<p$. So $G$ is of type (IV).

Case 3. Let $P=\langle x\rangle$, and let $Q$ be as in Lemma 2.6 (II) with $|Q|=q^{n}$. That is, $Q=\left\langle y, z \mid y^{q^{n-1}}=z^{q}=1, z^{-1} y z=y^{1+q^{n-2}}\right\rangle$, where $n \geq 3$, and $n \geq 4$ if $p=2$. In the similar way as Case 2, $y$ induces a power automorphism of order $q$ in $P$ and $\langle z\rangle \leq C_{G}(P)$. Furthermore, we have $x^{p}=1, y^{-1} x y=x^{i}$, where $p \equiv 1(\bmod q), i^{q} \equiv 1(\bmod p)$ with $1<i<p$. So $G$ is of type $(\mathrm{V})$.

(3) Assume that $G$ is supersolvable, $P$ is non-cyclic and $Q=\langle y\rangle$.

Since $G$ is supersolvable, we have easily that $P$ has at least two maximal subgroups which are normal in $G$ by applying Maschke's Theorem [10, 8.1.2] and Schreier's Refinement Theorem [10, 3.1.2]. By assumption, we further have that every maximal subgroup of $G$ has prime index, $\left\langle y^{q}\right\rangle=O_{q}(G)=Z(G), G$ has maximal subgroups $P\left\langle y^{q}\right\rangle^{g}$ and $P_{1}\langle y\rangle^{g}$, where $P_{1}$ is some maximal subgroup of $P$ which can permute with $\langle y\rangle^{g}$, and $g \in G$. If $G$ is a PST-group, then $P$ is abelian by applying Agrawal's Theorem in [1]. (A group $G$ is called a PSTgroup if $s$-quasinormality is a transitive relation in $G$, and Agrawal showed that $G$ is a solvable PST-group if and only if it has an abelian normal Hall subgroup $N$ on which $G$ acts by conjugation as power automorphisms and $G / N$ is a solvable PST-group.) By Lemma 2.5, $y$ induces a fixed-point-free power automorphism of order $q$ on $P$, i.e., $G$ is a SB-group, a contradiction. Clearly, all maximal subgroups of $G$ are PST-groups by assumption, and so $G$ is a minimal non-PST-group. An accurate examination of the list of minimal non-PST-groups in [11, Theorem 1], we have that either $G$ is of type (VI) with $j=1$ or $G$ is of type (VI) with $j \neq 1$.

(4) Assume that $G$ is minimal non-supersolvable and $Q$ is cyclic.

Denote $M=P_{3} Q \lessdot G$, where $P_{3}$ is a Sylow $p$-subgroup of $M$. By $\left[P_{3}, Q\right] \leq$ $P \cap P_{3} Q=P_{3}$, we have $N_{G}\left(P_{3}\right) \geq P_{3} Q=M$. On the other hand, $N_{P}\left(P_{3}\right)>P_{3}$, so $P_{3}$ is normal in $G$. By Lemma 2.2 and the maximality of $M, P_{3}=\Phi(P)$ is a Sylow $p$-subgroup of $M$. Thus, we prove that $\Phi(P)$ is a Sylow $p$-subgroup of $M$ if $Q \leq M \lessdot G$.

Case 1. If $G$ is also a minimal non-nilpotent group, then $G$ is of either type (VII) or type (VIII) by [3, Theorem 3].

Case 2. If $G$ is not a minimal non-nilpotent group and $P$ is abelian, by applying $[2$, Theorem 9,10$]$, we assume that $G=P Q$, where $P=\left\langle a_{0}, a_{1}, \ldots\right.$, $\left.a_{q-1}\right\rangle$ is an elementary abelian $p$-group of order $p^{q}, Q=\langle y\rangle$ is cyclic of order $q^{r}, q^{f}$ is the highest power of $q$ dividing $p-1$ and $r>f \geq 1$. The action of $Q$ on $P$ is defined by $a_{j}^{y}=a_{j+1}$ for $0 \leq j<q-1$ and $a_{q-1}^{y}=a_{0}^{i}$, where $i$ is a primitive $q^{f}$-th root of unity modulo $p$. 
By assumption, $P\left\langle y^{q}\right\rangle$ is a SB-group, and so $y^{q}$ induces a power automorphism of order $q$ in $P$ by Lemma 2.3. Hence $a_{j}^{y^{q^{2}}}=a_{j}^{i^{q}}=a_{j}, a_{q-1}^{y^{q^{2}}}=a_{q-1}^{i^{q}}=$ $a_{q-1}$. Thus, $i^{q} \equiv 1(\bmod p)$ and $f=1$, so $G$ is of type (IX).

Case 3. Assume that $G$ is not a minimal non-nilpotent group and $P$ is nonabelian. By $\left[2\right.$, Theorem 9, 10], we assume that $G=P Q$ such that $P=\left\langle a_{0}, a_{1}\right\rangle$ is an extraspecial group of order $p^{3}$ with the exponent $p, Q=\langle y\rangle$ is a cyclic group of order $2^{r}$ with $2^{f}$ the largest power of 2 dividing $p-1$ and $r>f \geq 1$, and $a_{0}^{y}=a_{1}$ and $a_{1}^{y}=a_{0}^{i} x$, where $x \in\left\langle\left[a_{0}, a_{1}\right]\right\rangle$ and $i$ is a primitive $2^{f}$-th root of unity modulo $p$.

By assumption, all subgroups of $P\left\langle y^{2}\right\rangle$ are SB-groups. If $P\left\langle y^{2}\right\rangle \neq P \times\left\langle y^{2}\right\rangle$, then by Lemma 2.3, $P$ is abelian, a contradiction. Hence $P\left\langle y^{2}\right\rangle=P \times\left\langle y^{2}\right\rangle$ and $a_{0}^{y^{2}}=a_{1}^{y}=a_{0}^{i} x=a_{0}$, which implies that $x=1$ and $i \equiv 1(\bmod p)$, a contradiction. So $G$ is not of the type as above.

Next we discuss the case that $|\pi(G)|$ has exactly three prime divisors.

By Lemma 2.4, we can assume that $G=P Q R$, where $P \in \operatorname{Syl}_{p}(G), Q \in$ $\operatorname{Syl}_{q}(G), R \in \operatorname{Syl}_{r}(G)$, and $p>q>r$. If $R$ is non-cyclic, then $P R$ and $Q R$ are all nilpotent by Lemma 2.3. Hence $R$ is normal in $G$. Let $R_{1}$ be an arbitrary maximal subgroup of $R$. Then $P Q R_{1}$ is a SB-group and hence $P Q R_{1}$ is nilpotent by Lemma 2.3 , which implies that $G$ itself is nilpotent, a contradiction. Thus, we have that $R$ is cyclic and $P Q \unlhd G$. By assumption, $P Q$ is either a nilpotent group or a group of the type (2) in Lemma 2.3 by Lemma 2.3.

Case 1. Assume that $P Q=P \times Q, P R=P \times R$ and $Q R=Q \times R$. Then $G$ is nilpotent, a contradiction.

Case 2. Assume that $P Q=P \times Q, P R=P \times R$ and $Q R=Q \rtimes R$. By Lemma 2.3, $Q$ is an abelian $q$-group and $R$ is cyclic. If $|P| \neq p$, then $P_{1} Q R$ is nilpotent by Lemma 2.3 again, where $1<P_{1}<P$, a contradiction. Hence $|P|=p$. Similarly, $|Q|=q$ and $G$ is a metacyclic group.

Therefore, we first assume that $G=\left\langle a, b \mid a^{p q}=b^{r^{m}}=1, b^{-1} a b=a^{i}\right\rangle$, where $i^{r^{m}} \equiv 1(\bmod p q), q \equiv 1(\bmod r), 1<i<p q$ and $\left(p q, r^{m}(i-1)\right)=1$. By assumption, $\left(a^{q}\right)^{b}=a^{i q}=a^{q}$, and so $i \equiv 1(\bmod p)$, a contradiction.

Next we assume that $G=\left\langle a, b \mid a^{q}=b^{p r^{m}}=1, b^{-1} a b=a^{i}\right\rangle$, where $i^{p r^{m}} \equiv$ $1(\bmod q), q \equiv 1(\bmod r), 1<i<q$ and $\left(q, p r^{m}(i-1)\right)=1$. Furthermore, $a^{b^{r}}=a^{i^{r}}=a$, and $a^{b^{p}}=a^{i^{p}} \neq a$. Hence $i^{r} \equiv 1(\bmod q), i^{p} \not \equiv 1(\bmod q)$, and $G$ is isomorphic to type (X).

Case 3. Assume that $P Q=P \times Q, P R=P \rtimes R$ and $Q R=Q \times R$. With similar arguments as above, we have $|P|=p,|Q|=q, R$ is cyclic.

Assume that $G=\left\langle a, b \mid a^{p q}=b^{r^{m}}=1, b^{-1} a b=a^{i}\right\rangle$, where $i^{r^{m}} \equiv$ $1(\bmod p q), p \equiv 1(\bmod r), 1<i<p q$ and $\left(p q, r^{m}(i-1)\right)=1$. However, $\left(a^{p}\right)^{b}=a^{i p}=a^{p}$, and so $i \equiv 1(\bmod q)$, a contradiction. 
Now we can assume that $G=\left\langle a, b \mid a^{p}=b^{q r^{m}}=1, b^{-1} a b=a^{i}\right\rangle$, where $i^{q r^{m}} \equiv 1(\bmod p), p \equiv 1(\bmod r), 1<i<p$ and $\left(p, q r^{m}(i-1)\right)=1$. Furthermore, $a^{b^{r}}=a^{i^{r}}=a$, and $a^{b^{q}}=a^{i^{q}} \neq a$. Hence $i^{r} \equiv 1(\bmod p), i^{q} \not \equiv 1(\bmod p)$, and $G$ is of type (X).

Case 4. Assume that $P Q=P \times Q, P R=P \rtimes R$ and $Q R=Q \rtimes R$. If $|P|>p$, then let $x$ be an element of $P$ with $x \neq 1, y \in Q$, and $z \in R$. By assumption, we have $(x y)^{z}=(x y)^{j}, x^{z}=x^{k}, y^{z}=y^{l}$ for some positive integers $j, k$ and $l$. Hence $(x y)^{z}=x^{j} y^{j}=x^{k} y^{l}=x^{z} y^{z}$. Furthermore, $x^{z}=x^{j}, y^{z}=y^{j}$, which implies that $R$ induces a fixed-point-free power automorphism of order $r$ on $P Q$, a contradiction. Hence $|P|=p$. Similarly, $|Q|=q$. Thus, $G$ is a metacyclic group. We only can assume that $G=\left\langle a, b \mid a^{p q}=b^{r^{m}}=1, b^{-1} a b=a^{i}\right\rangle$, where $i^{r^{m}} \equiv 1(\bmod p q), p \equiv 1(\bmod r), q \equiv 1(\bmod r), 1<i<p q$ and $\left(p q, r^{m}(i-1)\right)=1$. However, $G$ is a SB-group, a contradiction.

Case 5. Assume that $P Q=P \rtimes Q, P R=P \times R, Q R=Q \times R$. With similar arguments as above, $|P|=p,|R|=r$ and $Q$ is cyclic with $|Q|=q^{m}$.

We first assume that $G=\left\langle a, b \mid a^{p}=b^{q^{m} r}=1, b^{-1} a b=a^{i}\right\rangle$, where $i^{q^{m} r} \equiv$ $1(\bmod p), p \equiv 1(\bmod q), 1<i<p$ and $\left(p, q^{m} r(i-1)\right)=1$. Furthermore, $a^{b^{r}}=a^{i^{r}} \neq a$ and $a^{b^{q}}=a^{i^{q}}=a$. Hence $i^{r} \not \equiv 1(\bmod p), i^{q} \equiv 1(\bmod p)$, and $G$ is of type (XI).

Next we assume that $G=\left\langle a, b \mid a^{p r}=b^{q^{m}}=1, b^{-1} a b=a^{i}\right\rangle$, where $i^{q^{m}} \equiv$ $1(\bmod p r), p \equiv 1(\bmod q), 1<i<p r$ and $\left(p r, q^{m}(i-1)\right)=1$. Furthermore, $\left(a^{p}\right)^{b}=a^{i p}=a^{p}$, and so $i \equiv 1(\bmod r)$, a contradiction.

Case 6. Assume that $P Q=P \rtimes Q, P R=P \times R, Q R=Q \rtimes R$. With similar arguments as above, $|P|=p,|Q|=q, R$ is a cyclic subgroup of order $r^{m}$, and $G$ is a metacyclic group. This contradicts the assumption.

Case 7. Assume that $P Q=P \rtimes Q, P R=P \rtimes R, Q R=Q \times R$. With similar arguments as above, $|P|=p,|Q|=q,|R|=r$. We assume that $G=\left\langle a, b \mid a^{p}=b^{q r}=1, b^{-1} a b=a^{i}\right\rangle$, where $i^{q r} \equiv 1(\bmod p), p \equiv 1(\bmod q)$, $p \equiv 1(\bmod r), 1<i<p,(p, q r(i-1))=1$. Clearly, $i^{q} \not \equiv 1(\bmod p)$ and $i^{r} \not \equiv 1(\bmod p)$. So $G$ is of type (XII).

Case 8. Assume that $P Q=P \rtimes Q, P R=P \rtimes R, Q R=Q \rtimes R$. With similar arguments as above, $G$ is a metacyclic group, a contradiction. Hence $G$ does not exists.

Conversely, it is clear that a group which belongs to types (I), (II), (X)(XII) is a minimal non-SB-group.

For type (III), $G$ is not nilpotent, and all Sylow 2-subgroups of $G$ are noncyclic, $G$ is not a SB-group by Lemma 2.3. Since $\left\langle x^{2 p}\right\rangle=Z(G)$ is the unique subgroup of $G$ of order 2 and $G$ has maximal groups of orders $4 p$ and 8 , we have that $G$ is a minimal non-SB-group.

For type (IV), it is clear that $G$ is not a SB-group. With similar arguments used in Case 2 of (2), $G$ has maximal subgroups $P H^{g}, P K_{0}^{g}, P K_{s}^{g}$ and $Q^{g}$, where $g \in G, K_{0}=\left\langle y^{q}, z\right\rangle, K_{s}=\left\langle z y^{s}\right\rangle$ with $s=1, \ldots, q-1$. Since $\left\langle y^{q}\right\rangle$ 
is normal in $G$ and $z \in Z(G)$, we have that all proper subgroups of $G$ are SB-groups. Therefore, $G$ is a minimal non-SB-group.

For type (V), in the similar way as type (IV), we conclude that $G$ is a minimal non-SB-group easily.

For type (VI), we conclude easily that $G$ has maximal subgroups $\langle a\rangle Q^{g}$, $\langle b\rangle Q^{g}$ and $Q^{g}$, where $g \in G$. Clearly, $G$ is a minimal non-SB-group.

For types (VII) and (VIII), since $G$ is minimal non-nilpotent, $G$ is nonsupersolvable by [15, Theorem 2.8]. In view of Lemma 2.1, $G$ is not a SB-group, and so $G$ is a minimal non-SB-group.

For type (IX), since $G$ is minimal non-supersolvable, $G$ is not a SB-group by Lemma 2.1. Similar arguments used in (4) lead to the fact that $G$ has maximal subgroups $P\left\langle y^{q}\right\rangle^{u}$ and $Q^{u}$, where $u \in G$. Clearly, $G$ is a minimal non-SB-group.

Remark 3.1. By applying Lemma 2.7, Lemma 2.8 and Lemma 2.9, similar procedure to the proof of Theorem 1.1, we easily obtain Theorem 1.2 and Theorem 1.3, so we omit their proofs.

Acknowledgements. The authors express their deep sense for gratitude to the referees for their valuable comments to polish this paper.

\section{References}

[1] R. K. Agrawal, Finite groups whose subnormal subgroups permute with all Sylow subgroups, Proc. Amer. Math. Soc. 47 (1975), no. 1, 77-83.

[2] A. Ballester-Bolinches and R. Esteban-Romero, On minimal non-supersoluble groups, Rev. Mat. Iberoamericana 23 (2007), no. 1, 127-142.

[3] A. Ballester-Bolinches, R. Esteban-Romero, and D. J. S. Robinson, On finite minimal non-nilpotent groups, Proc. Amer. Math. Soc. 133 (2005), no. 12, 3455-3462.

[4] K. Doerk, Minimal nicht überauflösbare, endliche Gruppen, Math. Z. 91 (1966), 198205.

[5] A. Fattahi, Groups with only normal and abnormal subgroups, J. Algebra 28 (1974), 15-19.

[6] P. F. Guo and X. Y. Guo, On minimal non-MSN-groups, Front. Math. China 6 (2011), no. 5, 847-854.

[7] X. Y. Guo, K. P. Shum, and A. Ballester-Bolinches, On complemented minimal subgroups in finite groups, J. Group Theory 6 (2003), no. 2, 159-167.

[8] Z. J. Han, G. Y. Chen, and W. Zhou, On minimal non-NSN-groups, J. Korean Math. Soc. 50 (2013), no. 3, 579-589.

[9] S. R. Li, On minimal non-PE-groups, J. Pure Appl. Algebra 132 (1998), no. 2, 149-158.

[10] D. J. S. Robinson, A Course in the Theory of Groups, Springer-Verlag, New YorkHeidelberg-Berlin, 1982.

[11] Minimality and Sylow-permutability in locally finite groups, Ukrainian Math. J. 54 (2002), no. 6, 1038-1048.

[12] O. J. Schmidt, Über Gruppen, deren sämtliche Teiler spezielle Gruppen sind, Mat. Sbornik 31 (1924), 366-372.

[13] Z. C. Shen, S. R. Li, and W. J. Shi, Finite groups all of whose second maximal subgroups are PSC-groups, J. Algebra Appl. 8 (2009), no. 2, 229-242.

[14] J. G. Thompson, Nonsolvable finite groups all of whose local subgroups are solvable, Bull. Amer. Math. Soc. 74 (1968), 383-437. 
[15] M. Y. Xu and Q. H. Zhang, On conjugate-permutable subgroups of a finite group, Algebra Colloq. 12 (2005), no. 4, 669-676.

[16] Q. H. Zhang, Finite groups with only s-quasinormal and abnormal subgroups, Northeast. Math. J. 14 (1998), no. 1, 41-46.

[17] _ s-semipermutability and abnormality in finite groups, Comm. Algebra 27 (1999), no. 9, 4515-4524.

[18] Y. Zhao, A note on the structure of Quasi-Hamilton groups, J. Engineering Math. 9 (1992), no. 4, 119-121.

Pengfei Guo

School of Mathematics And Information EngineERING

Lianyungang Normal College

LiAnyungang 222006, P. R. China

AND

Department of Civil and Architectural Engineering

City University of Hong Kong

Hong Kong 999077, P. R. ChinA

E-mail address: guopf999@163.com

JUNXIN WANG

Department of Mathematics

Shanxi University of Finance And Economics

Taiyuan 030006, P. R. China

E-mail address: wangjunxin660712@163.com

HAILIANG ZHANG

Department of Mathematics

ZheJiang OCEAN University

Zhoushan 316000, P. R. China

E-mail address: hlzhang@zjou.edu.cn 